\title{
Pelvic Floor Muscle Training Included in a Pregnancy Exercise Program Is Effective in Primary Prevention of Urinary Incontinence: A Randomized Controlled Trial
}

\author{
Mireia Pelaez, ${ }^{1 *}$ Silvia Gonzalez-Cerron, ${ }^{2}$ Rocío Montejo, ${ }^{2}$ and Rubén Barakat ${ }^{1}$ \\ ${ }^{1}$ Faculty of Physical Activity and Sport Sciences-INEF, Technical University of Madrid-UPM, Madrid, Spain \\ ${ }^{2}$ Universitarian Hospital of Fuenlabrada, Madrid, Spain
}

\begin{abstract}
Aims: To investigate the effect of pelvic floor muscle training (PFMT) taught in a general exercise class during pregnancy on the prevention of urinary incontinence (UI) in nulliparous continent pregnant women. Methods: This was a unicenter two armed randomized controlled trial. One hundred sixty-nine women were randomized by a central computer system to an exercise group (EG) (exercise class including PFMT) $(\mathrm{n}=73$ ) or a control group (CG) (n = 96). $10.1 \%$ loss to follow-up: 10 from EG and 7 from CG. The intervention consisted of 70-75 sessions (22 weeks, three times per week, 55-60 min/session including $10 \mathrm{~min}$ of PFMT). The CG received usual care (which included follow up by midwifes including information about PFMT). Questions on prevalence and degree of UI were posed before (week 1014) and after intervention (week 36-39) using the International Consultation on Incontinence Questionnaire-Urinary Incontinence Short Form (ICIQ-UI SF). Results: At the end of the intervention, there was a statistically significant difference in favor of the EG. Reported frequency of UI [Never: CG: 54/60.7\%, EG: 60/95.2\% $(P<0.001)$ ]. Amount of leakage [None: CG: 45/60.7\%, EG: 60/95.2\% ( $P<0.001)$ ]. There was also a statistically significant difference in ICIQUI SF Score between groups after the intervention period [CG: 2.7 (SD 4.1), EG: 0.2 (SD 1.2) $(P<0.001)$ ]. The estimated effect size was 0.8. Conclusion: PFMT taught in a general exercise class three times per week for at least 22 weeks, without former assessment of ability to perform a correct contraction was effective in primary prevention of UI in primiparous pregnant women. Neurourol. Urodynam. (c) 2013 Wiley Periodicals, Inc.
\end{abstract}

Key words: gestation; nulliparous; physical exercise; physical therapy; prevention

\section{INTRODUCTION}

Urinary incontinence (UI) is a well recognized health problem that significantly reduces the quality of life and affects women of all ages. ${ }^{1-3}$ Pregnancy and childbirth are considered to be important risk factors in the development and the worsening of UI. ${ }^{1,4}$

Pelvic floor muscle training (PFMT) is an effective technique in the prevention and treatment of UI in the general population $^{4}$ and its effectiveness has also been found during pregnancy. ${ }^{1}$ Moreover, based on different studies, it has been found to be as effective as other treatments ${ }^{5,6}$ and according to a review by Cochrane, it should be included in the first line of conservative management and of primary prevention of UI. $^{7}$

In most studies, PMFT is conducted by physiotherapists and its efficacy when taught by other professionals such as fitness instructors has not been found. ${ }^{8}$ To quote $B \varnothing$ and Haakstad ${ }^{8}$ it would be less time-consuming, more cost-effective and possibly more motivating if PFMT could be taught in a group setting by fitness instructors. However the professional who should design and lead the session must meet different requirements. A Physical Activity and Sport Sciences graduate has both the theoretical and practical knowledge to design exercise programs taking into account the training principles, and moreover has a pedagological background which could enhance motivation and long-term learning.

We hypothesized that PFMT taught by a Physical Activity and Sport Sciences graduate and included in a pregnancy exercise program is effective in primary prevention of UI.

\section{MATERIALS AND METHODS}

Design

This was a unicenter, two armed, open label, randomized controlled trial.

\section{Participants}

Pregnant women attending the local hospital for antenatal care were asked to participate in the study. Recruitment took place between October 2009 and October 2011, and final data collection finished on June 2011. Inclusion criteria were: being healthy and primiparous pregnant with singleton fetus, being in gestational week 10-14, not suffering from UI, able to communicate in Spanish and able to give informed written consent. Exclusion criteria were: planning not to give birth in Fuenlabrada University Hospital and any contraindication according to the American College of Obstetricians and Gynecologists Guidelines. ${ }^{9}$ One hundred sixty nine women were included in the study. It was approved by the Ethical

\section{Conflict of interest: none.}

Mickey Karram led the peer-review process as the Associate Editor responsible for the paper

${ }^{*}$ Correspondence to: Mireia Pelaez, PhD, Faculty of Physical Activity and Sport Sciences-INEF, Technical University of Madrid-UPM, Martín Fierro, 7, 28040 Madrid, Spain.E-mail: mireiapelaez@gmail.com

Received 25 July 2012; Accepted 14 January 2013

Published online in Wiley Online Library

(wileyonlinelibrary.com)

DOI 10.1002/nau.22381 
Committee of Fuenlabrada University Hospital (Madrid, Spain) and followed the ethical guidelines outlined in the Declaration of Helsinki (last modified in 2008). All women provided written informed consent. The study is listed in ClinicalTRials.gov (NCT 01578369).

\section{Intervention}

Women in the exercise group (EG) were asked to participate in a structured exercise program for at least 22 weeks (14-36 weeks of gestation). The program consisted of 70-78 group sessions (8-12 women). The sessions were held three times per week and were 55-60 min in duration. The sessions were performed in the hospital, in a well-lit room under favorable environmental conditions (altitude $600 \mathrm{~m}$; temperature, 19$21^{\circ} \mathrm{C}$; humidity, $50-60 \%$ ).

Each session consisted of 8 min of warm-up; 30 min of low impact aerobics (performing different choreographies) including $10 \mathrm{~min}$ of general strength training (e.g., core muscles, pectoralis, gluteus, quadriceps, calves, biceps); $10 \mathrm{~min}$ of PFMT and $7 \mathrm{~min}$ of cool down, which included stretching, relaxation or massage. All subjects used a heart rate monitor (Accurex Plus, Polar Electro OY, Finland) during the training sessions to control the exercise intensity $(65-70 \%$ age predicted maximum heart rate value). The Borg Rating of Perceived Exertion Scale was also used to guide exercise intensity (12-14 "somewhat hard"). ${ }^{10}$

The whole session, including PFMT was designed and taught by a Physical Activity and Sport Sciences graduate. At the beginning of the program, women received information of anatomy and function of the PFM and why the muscles may prevent and treat the UI. At first, women learned to perceive their pelvic floor, and then learned how to do adequate contractions. There was no former assessment of ability to perform a correct contraction or any measurement of pelvic floor muscle strength. Women were asked to test themselves at home by trying to stop the flow while urinating, touching the vaginal and anal openings and using a mirror to see if they could squeeze around the pelvic openings and upward lift.

The PFMT followed a progression, starting with one set of eight contractions and increasing the number of total contractions to one hundred, divided in different sets of slow (6 sec) and fast contractions (five contractions as fast as possible). To increase motivation and abilities, we used different exercises (Fig. 1), positions and sometimes music to follow the beat. Women were encouraged to perform 100 contractions distributed in different sets every day.

To enhance motivation and adherence, group dynamics and exercises in pairs were included. Talking about needs and feelings were also allowed and encouraged.

Women in the control group (CG) received usual care (which included follow up by midwifes including information about PFMT) and were not asked not to train their pelvic floor muscles.

\section{Randomization}

A statistical randomization computer program was used to perform a simple randomization to allocate women into each group.

\section{Outcome Measures}

The primary outcomes were (i) the reported frequency, (ii) amount, and (iii) impact on daily life of UI. These outcomes were measured by the validated International Consultation on Incontinence Questionnaire-Urinary Incontinence Short Form (ICIO-UI SF). ${ }^{11}$ Women completed the forms at the end of

\begin{tabular}{|l}
\hline Examples of some of the proposed exercises \\
\hline - Contract all PFM trying to close and lift the openings. \\
- Contract all PFM while inhale and relax while exhale. \\
- Contract all PFM while exhale and relax while inhale. \\
- Imagine you want your pubis and coccyx bones get closer contracting your PFM in this \\
- direction. \\
- Imagine you want your ischial tuberosities get closer contracting your PFM in this \\
- Contract all PFM with a $25 \%$ of maximal strength, increase until $50 \%$ and $100 \%$ and \\
- - Try to contract only urethra area, add vagina, add anal area. Relax. \\
- Try to contract only anal area, add vagina, add urethra area. Relax. \\
- Contract PFM, cough keeping it contracted and relax. \\
- Contract PFM following the beat of music, doing different rhythms. \\
\hline
\end{tabular}

Fig. 1. Examples of some of the proposed exercises in pelvic floor muscle training. PFM, pelvic floor muscles. 
intervention (between 36 and 40 weeks of gestation). Before intervention, women were interviewed to provide maternal characteristics such as education level or pre-pregnancy physical activity habits. To enhance the quality of measurements, the three interviewers were trained and informed about how to make women feel comfortable talking about their UI.

\section{Power Calculations}

To estimate the sample size, a 95\% confidence level, $80 \%$ statistical power, and a $20 \%$ of losses were accepted. Assuming, in the usual care situation, a prevalence of $39 \%$ of women affected by UI at the end of gestation (week 36) ( $^{3}$ and estimating a reduction of $20 \%$ of women affected by UI in the treatment group, it was considered that 156 women should be included.

\section{Statistical Analyses}

Background variables are given as frequencies and percentages, and means with standard deviations (SD). Chi-squared and Mann-Whitney tests were used to analyze differences in categorical variables and $t$-test for quantitative variables. $P$ values $<0.05$ were considered to be statistically significant.
Effect size was also calculated on ICIO-Score using $t$-stats and sample size.

\section{RESULTS}

Initially, 169 women were included in the study. Data from 152 women were analyzed (10.1\% loss to follow-up: 10 from the EG and 7 from the CG). Figure 2 shows the study flow chart with the numbers of drop-outs and their reasons. All women included on analysis attended at least $80 \%$ of $70-78$ exercise sessions during at least 22 weeks.

Background variables at baseline are shown in Table I. There were no differences between groups in mean age, pre-pregnancy BMI, education, and pre-pregnancy physical exercise habits at baseline.

At the end of intervention (week 36 of pregnancy) there were statistically significant differences in favor of the EG as shown in Table II). $95.2 \%$ of women in the EG reported no leakage versus a $60.7 \%$ of women in the CG. Only women of CG had a frequency of leakage more than of once a week (17/19.1\%). The amount of leakage reported by incontinent women in the EG is referred as a "a small amount" (3/4.8\%) but in the CG, women reported "a small amount" (27/30.6\%), "a moderate amount" (5/ $5.6 \%)$, and even "a large amount" (3/3.4\%).

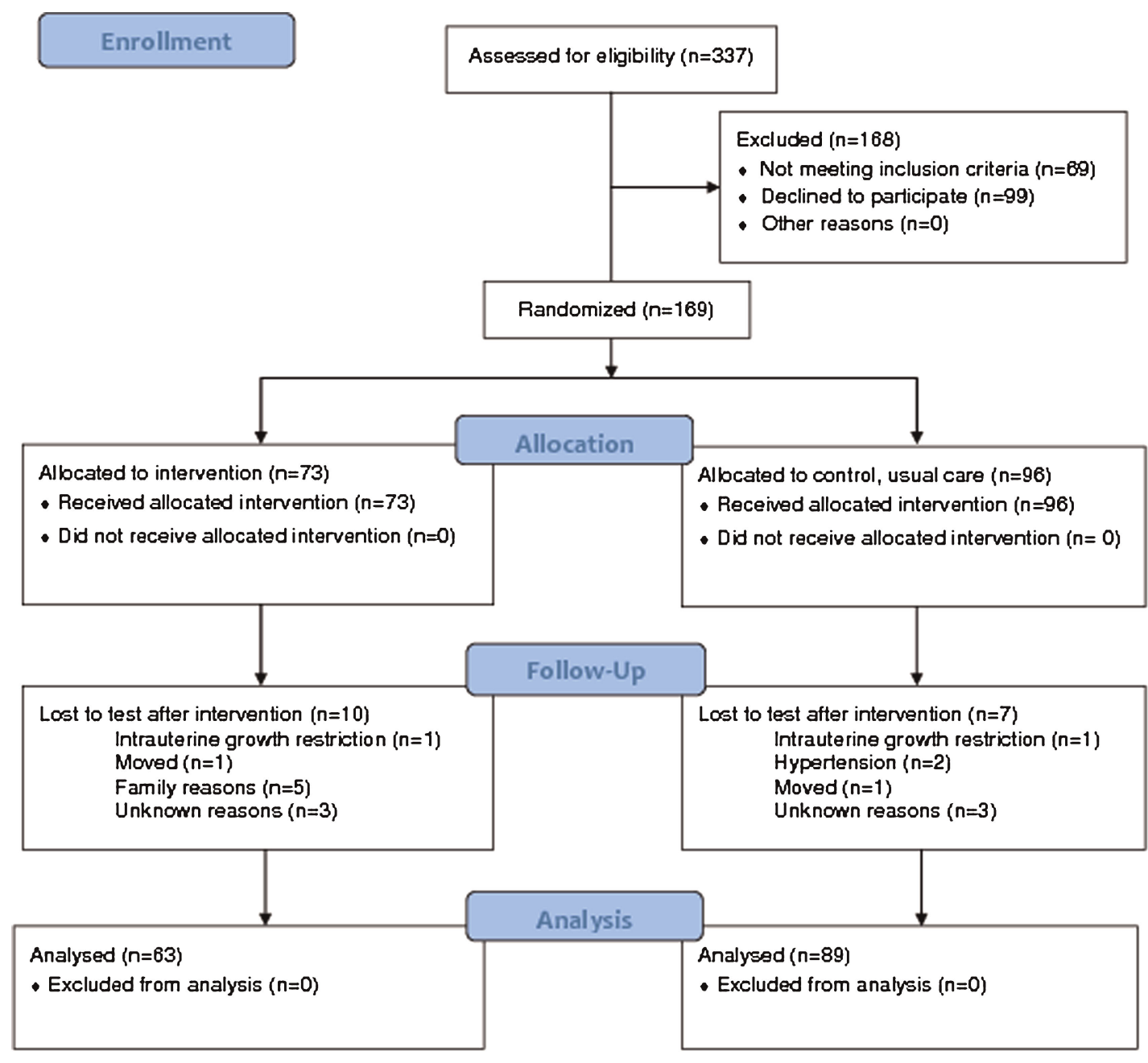

Fig. 2. Study flow chart with the number of drop-outs and their reasons. 
TABLE I. Background Variables Before Intervention in the Exercise and Control Groups

\begin{tabular}{llll}
\hline & $\begin{array}{c}\text { Exercise } \\
(\mathrm{n}=63)\end{array}$ & $\begin{array}{c}\text { Control } \\
(\mathrm{n}=89)\end{array}$ & $P$-value \\
\hline Age (years) & $29.9(3.3)$ & $29.1(4.5)$ & 0.23 \\
$\begin{array}{l}\text { Pre-pregnancy BMI } \\
\text { Educational level }\end{array}$ & $23.6(4.3)$ & $22.7(3.8)$ & 0.23 \\
$\quad$ College & $23 / 37.1$ & $23 / 25.8$ & 0.07 \\
$\quad$ High school & $29 / 46.8$ & $38 / 42.7$ & \\
$\quad$ <High school & $10 / 16.1$ & $28 / 31.5$ & \\
$\begin{array}{l}\text { Pre-pregnancy PA } \\
\quad \text { Inactive }\end{array}$ & $33 / 53.2$ & $56 / 62.9$ & 0.15 \\
$\quad$ Active & $29 / 46.8$ & $33 / 37.1$ & \\
\hline
\end{tabular}

BMI, body mass index; PA, physical habits.

Mean with standard deviation (SD) and numbers (n) with percentages (\%) ( $\mathrm{n}=152)$

In regards to how much leaking affects their daily lives, the mean in the CG was higher than in the EG (0.9 (SD 1.8) vs. 0.1 (SD 0.6), respectively. Finally, ICIO-UI SF Score shows statistically significant differences between the groups and the estimated effect size was 0.8 .

\section{DISCUSSION}

The major finding of the present study was that PFMT included in a pregnancy exercise program, with three sessions per week over the course of at least 22 weeks, is effective in primary prevention of UI in pregnancy, without individual assessment of the ability to contract the pelvic floor muscles.

The strengths of the present study include the use of an RCT design, high adherence and the implementation of an exercise program following ACOG recommendations, conducted by a Physical Activity and Sport Sciences graduate. Some of the limitations of the study are: no pad test, no assessment of PFM strength and the non-blinded design.

The prevalence rates of UI in the CG were very similar to those that we took into account to estimate the sample size. We assumed a prevalence of $39 \%$ of women affected by UI at the end of gestation (week 36$)^{3}$ and in our study we found that a $39.3 \%$ of women in the CG were affected by UI, which

TABLE II. Results From ICIO-UI SF Questionnaire After Intervention in Exercise and Control Groups

\begin{tabular}{lccc}
\hline & $\begin{array}{c}\text { Exercise } \\
(\mathrm{n}=63)\end{array}$ & $\begin{array}{c}\text { Control } \\
(\mathrm{n}=89)\end{array}$ & P-value \\
\hline & & & \\
Reported frequency of UI & $60 / 95.2$ & $54 / 60.7$ & 0.0001 \\
Never & $3 / 4.8$ & $18 / 20.2$ & \\
Once a week & $0 / 0.0$ & $9 / 10.1$ & \\
2-3 times per week & $0 / 0.0$ & $7 / 7.9$ & \\
Once a day & $0 / 0.0$ & $1 / 1.1$ & \\
Several times per day & & & \\
Amount of leakage & $60 / 95.2$ & $54 / 60.7$ & \\
None & $3 / 4.8$ & $27 / 30.3$ & \\
A small amount & $0 / 0.0$ & $5 / 5.6$ & 0.0001 \\
A moderate amount & $0 / 0.0$ & $3 / 3.4$ & \\
A large amount & $0.10(0.64)$ & $0.97(1.8)$ & \\
How much does leaking & $0.24(1.2)$ & $2.66(4.1)$ & 0.0001 \\
affect daily life & & & \\
ICIO-Score & &
\end{tabular}

Mean with standard deviation (SD) and numbers (n) with percentages (\%) $(\mathrm{n}=152)$. was in accordance with these data. We also estimated a reduction of $20 \%$ of women affected by UI in the EG, and the reduction obtained was higher: a $34.5 \%$.

The reduction of UI found in the EG is in accordance with other studies in which PFMT is lead by physiotherapists. Mørkved et al. ${ }^{1}$ found $16 \%$ difference in the prevalence of UI in favor of the training group in pregnant nulliparous women. However this study included incontinent women $32 \%$ and $31 \%$ in training and CG respectively) at baseline and there was only one supervised session per week. Stafne et al. ${ }^{12}$ found fewer women in the intervention group reporting any weekly UI (11\% vs. 19\%) at the end of intervention (32-36 weeks of gestation). Only one weekly group session was led by physiotherapists during the 12-week exercise program.

To date, we have only found two studies in which PFMT was not conducted by physiotherapists. The first one by Brubaker et al. ${ }^{13}$ shows the feasibility of a pelvic fitness and education class for affected and non pregnant women. The women in this study trained twice a week for 11 weeks. The results showed statistically and clinically significant improvement in pelvic floor and sexual function. The second study, by $\mathrm{B} \varnothing$ and Haakstad ${ }^{8}$ had a RCT design and PFMT were led by certified aerobics instructors. No effect of PFMT was found and that is in contrast with our results.

We found common points in $\mathrm{B} \varnothing$ and Haakstad ${ }^{8}$ and the present study. An important point is that there was no former assessment of ability to perform a correct contraction. The second common point was the exercise program structure. Perhaps, one of the main differences was the adherence to the program. Only $40 \%$ of women in the aforementioned study attended at least $80 \%$ of exercise sessions. In our study, all women included on analysis (100\%) attended at least $80 \%$ of sessions. Other differences were the length of the program (12 weeks vs. 22 in the present study) and the total number of pelvic floor muscle contractions per day (36 vs. 100 respectively). Furthermore, in the $\mathrm{B} \phi$ et al. study, there were incontinent women at baseline (26.9\% in the EG and $20.7 \%$ in the CG).

These results show the importance that a Physical Activity and Sport Sciences graduate design and lead the exercise programs to make them effective and motivating for the pregnant population. They also suggest that the three times per week frequency is well accepted. Although there were positive results without individual assessment, it may well be that the effect would have been far stronger if there had been individual assessment of the ability to contract the pelvic floor muscles.

Usual care for pregnant women includes 8-12 sessions with a midwife in which women learn about the pelvic floor as well as other issues concerning labor, breastfeeding and child care, but there is no standardized protocol. These results suggest the need for accompaniment, monitoring and motivation through the whole gestation period for PFMT to be effective and the importance of the development of multidisciplinary teams composed of different professionals to monitor and nurture pregnant women through gestation to ensure the highest quality of life as possible.

In future, it would be of interest to compare the prevalence of UI between groups 2 years after delivery as well as other aspects regarding the quality of life of these women; and to study the effectiveness of this type of program in the treatment of UI in affected women.

\section{CONCLUSION}

This study shows the effectiveness of PFMT included in a pregnancy exercise program on primary prevention of UI during pregnancy in nulliparous continent women. It also 
suggests the importance of both the program design and the role of a Physical Activity and Sport Sciences graduate in enhancing adherence to the program.

\section{ACKNOWLEDGMENTS}

The authors would like to acknowledge the technical assistance of the Gynecology and Obstetrics Service of Fuenlabrada University Hospital, the assistance of Ms. Marta de Rio and all participating woman.

\section{REFERENCES}

1. Mørkved S, B $\varnothing \mathrm{K}$, Schei B, et al. Pelvic floor muscle training during pregnancy to prevent urinary incontinence: A single-blind randomized controlled trial. Obstet Gynecol 2003;101:313-9.

2. Zhu L, Li L, Lang JH, et al. Prevalence and risk factors for peri- and postpartum urinary incontinence in primiparous women in China: A prospective longitudinal study. Int Urogynecol J 2012;23:563-72.

3. Solans-Domenech $M$, Sánchez E, Espuña-Pons $M$, et al. Urinary and ana incontinence during pregnancy and postpartum: Incidence, severity, and risk factors. Obstet Gynecol 2010;115:618-28.

4. Boyle R, Hay-Smith EJ, Cody JD, et al. Pelvic floor muscle training for preven tion and treatment of urinary and faecal incontinence in antenatal and postnatal women. Cochrane Database Syst Rev 2012;17:CD007471.
5. $\mathrm{B} \phi \mathrm{K}$, Talseth T, Holme I. Single blind, randomised controlled trial of pelvic floor exercises, electrical stimulation, vaginal cones, and no treatment in management of genuine stress incontinence in women. BMJ 1999;318: 487-93.

6. Castro RA, Arruda RM, Zanetti MR, et al. Single-blind, randomized, controlled trial of pelvic floor muscle training, electrical stimulation, vaginal cones, and no active treatment in the management of stress urinary incontinence. Clinics (Sao Paulo) 2008;63:465-72.

7. Hay-Smith EJ, B $\varnothing$ Berghmans LC, Hendriks HJ, et al. Pelvic floor muscle training for urinary incontinence in women. Cochrane Database Sys Rev 2007; CD001407.

8. $\mathrm{B} \phi \mathrm{K}$, Haakstad LA. Is pelvic floor muscle training effective when taught in a general fitness class in pregnancy? A randomised controlled trial. Physiotherapy 2011;97:190-5.

9. American College of Obstetricians and Gynecologists (ACOG). Exercise during pregnancy and the postpartum period. Committee Opinion 267. Obstet Gynecol 2002;99:171-3.

10. Artal R, O'Toole M, White S. Guidelines of the ACOG for exercise during pregnancy and postpartum period. Br J Sports Med 2003;37:6-1.

11. Avery K, Donovan J, Peters TJ, et al. ICIO: A brief and robust measure for evaluating the symptoms and impact of urinary incontinence. Neurourol Urodyn 2004;23:322-30

12. Stafne S, Salvensen KA, Romundstad $\mathrm{P}$, et al. Does regular exercise including pelvic floor muscle training prevent urinary and anal incontinence during pregnancy? A randomized controlled trial. BJOG 2012;119: 1270-80.

13. Brubaker L, Shott S, Tomezsko J, et al. Pelvic floor fitness using lay instructors. Obstet Gynecol 2008;111:1298-304. 